\title{
Fit to the task: using Canadian data for evidence-informed public health
}

\section{Angela Eykelbosh ${ }^{\text {** }}$ \\ The challenge of data gaps in public health}

aNational Collaborating Centre for Environmental Health and BC Centre for Disease Control, Vancouver, British Columbia.
Evidence-informed decision-making (EIDM) is the practice of integrating scientific research evidence with the many other competing social and political considerations that inform policy (National Collaborating Centre for Methods and Tools, 2012). However, decision-makers and those who participate in policy development are often confounded in this effort by data that are patchy, weak, or missing altogether. We must often reach for the "next best thing," typically data or studies from other demographically "comparable" nations, whom we assume to live, eat, work, and recreate in a manner closely similar to our Canadian population, under similar environmental conditions. This is problematic given that, within our own Canadian population, there is a wide disparity in factors influencing health, such as access to healthy foods, clean drinking water, and the presence of environmental contaminants.

Also at issue is how we use data. Although it may be useful to know that a given intervention had a certain effect in some other population (i.e., a static study), having access to detailed health data collected repeatedly over time (i.e., surveillance data) allows us to not only make comparisons amongst populations and track changes in health status, but also predict the health impacts of specific interventions and evaluate them after implementation. The aim of this short commentary is to highlight two large, cross-sectional, representative Canadian data sets that have great potential to inform public health policy; one such example is the use of research around the built environment.

\section{Canadian data sets}

Significant efforts have been made in recent years to build data resources that reflect our Canadian way of life and the environmental health challenges we face-data that are, in fact, fit to the task. Many of these data sets have been made available through the Government of Canada's Open Data Portal, which allows users to search for data sets by subject matter and key words. Among the many data sets already available, there are two largescale initiatives that have high value for understanding and addressing environmental health issues.

*Corresponding author: Angela Eykelbosh (email: Angela.Eykelbosh@ bccdc.ca)
Some readers may already be familiar with the Canadian Community Health Survey (CCHS), which was conducted every 2 years from 2001 to 2005, and then annually from 2007 until present. Since 2007, each cycle has collected data from approximately 65,000 Canadians, creating a very large sample that allows analysis to the level of the health region every 2 years. The general health component of the CCHS collects detailed information on self-reported health status, health care utilization, and health determinants that are informative to environmental health issues, as will be illustrated in the next section. In addition to the annual survey on general health, the CCHS also includes focused surveys on specific health issues, which are conducted every 3 years and collect information from approximately 35,000 people per cycle. Previous focused surveys have examined healthy aging, nutrition, and mental health.

The Canadian Health Measures Survey (CHMS) is a nationally representative human biomonitoring $(\mathrm{HBM})$ survey $^{1}$ that collects detailed health and exposure data on a biennial basis. By the end of its eight data collection cycles (2009-2023), the CHMS will comprise data on more than 40,000 Canadians and will contain large amounts of detailed health and exposure information highly relevant for environmental health research and policy. The strength of the CHMS data set is that it combines survey information with direct or objective measures. In the survey phase, participants are asked to provide details on health history, life satisfaction, diet, drinking water source, physical activity, active transport, hobbies, time spent outdoors, and their home and neighbourhood environments. This information is complemented by home environmental samples (tap water and indoor air), as well as a rigorous health and fitness assessments, eye and dental exams, and blood, urine and hair sample collection. Thus, the CHMS includes many of the topic areas examined by the CCHS, but is not reliant solely on self-reported measures. Up to cycle 6, it will also include data on 279 chemicals in blood and urine samples, including pesticides, metals and trace elements, persistent organic pollutants, environmental phenols, and several other classes of compounds. These chemicals were chosen as they are of known or emerging concern, as detailed in Canada's Chemical Management Plan (Government of Canada, 2012).

\footnotetext{
${ }^{1}$ Although we focus here on the CHMS, three other large-scale HBM initiatives worth mentioning are the First Nations Biomonitoring Initiative (FNBI) (AFN, 2013), the Maternal-Infant Research on Environmental Chemicals (MIREC) project (Government of Canada, 2019a), and the Northern Contaminants Program (Government of Canada, 2019b).
} 
The depth and detail of the CHMS make it incredibly informative to environmental health. However, because of the small number of collection sites per cycle, the CHMS data cannot be analyzed at the health region level. The smallest units that could potentially be analyzed, once all cycles are complete, are either provincial (BC, Ontario, and Quebec) or regional (Prairies, Atlantic Provinces). For a more thorough discussion of the CHMS and its relevance for environmental health, please see our recent review by Eykelbosh et al. (2018).

\section{Canadian data and the built environment}

Canadian data have the ability to show us even more than the health status of populations of interest or where intervention is most needed. These data can also be used to guide our policy decisions, through the use of tools and models that compare the effect of various interventions. They can also help us evaluate the success of certain interventions through meaningful comparison to other similar communities.

Numerous studies have already used the CCHS data set to understand how features of the built environment may affect physical health indicators, healthy behaviours, and mental health. Some examples of these include Pouliou and Elliot (2010), who found that variables such as land use mix, residential density, street connectivity, and walkability were individually negatively associated with body mass index. McMorris et al. (2015) used CCHS data to show that adults living in the greenest residential areas were more likely do physical activity in their leisure time. de Sa and Ardern (2014) found that people living in neighbourhoods with the highest density of intersections were more likely to walk or cycle for leisure, and that higher residential density was associated with active transport. These, and other examples, demonstrate the utility of the CCHS data set for understanding the potential influences of the built environment on human health.

However, the data can do more. Ulmer et al. (2015) used a predictive modelling tool to examine how redevelopment of the West Don lands in Toronto would affect health indicators, both for those living on the study site and in the surrounding neighbourhoods. By first establishing a relationship between built environment measures and health indicators obtained from the CCHS and other data sources, they were able to quantitatively estimate how the community plan would influence outcomes such as frequency of active transport, transit use, leisure walking, and potential decreases in body mass index. Even without quantitative estimates, this type of study is useful to demonstrate the trade-offs that occur as a result of planning decisions.

In a second example, Arnason et al. (2019) modelled the effects of transportation policy aimed at increasing active transport on the risk of diabetes, where diabetes risk was estimated from and validated using the CCHS data set. This allowed the researchers to understand the public health impact of specified active transport targets in terms of the number of diabetes cases avoided amongst commuters, for which the greatest impact was seen for adults over 45 years of age. Modelling studies such as this one allow planners and decision-makers to understand the effect of transportation policies (and associated infrastructure costs) in terms of human lives and avoided health care costs.
Relatively few studies have made use of the CHMS data to examine the built environment, and these have principally focused on the effect of neighbourhood walkability on selfreported and directly measured physical activity (Hajna et al., 2015; Thielman et al., 2015; Winters et al., 2015). However, the most recent cycle five data set includes new items on the neighbourhood environment, including dwelling types, destinations, proximity to transit, presence of sidewalks, access to low-cost recreation facilities, visual interest, perceived crime threat, and access to a vehicle. Because these data are directly linked to physical activity and health information, as well as mental health and satisfaction-with-life indicators, the CHMS data will provide insight into how investments in urban environments and transportation affect population health, albeit on a larger regional/ national (rather than municipal) scale.

\section{Leveraging partnerships to exploit Canadian data}

Data access and capacity are two of the greatest challenges in utilizing the large data sets describing our Canadian population. Unsurprisingly, members of the public are hesitant to share their personal information, their health data, and their tissues unless it can be promised that their data will be kept secure. That need for privacy is at the heart of Statistics Canada's data access policies. Those wishing to access the CHMS or CCHS microdata must submit an application detailing their research objectives and demonstrating the scientific merit of the project. Applicants must go through a security check and some users may be required to pay an access fee. Once approved, data can only be accessed within a Research Data Centre, which have been established in cities across Canada and are staffed by Statistics Canada analysts (Statistics Canada, 2019).

Despite these barriers, a recent review of CHMS data usage has shown how universities, provincial organizations, and federal agencies have successfully partnered to take advantage of the data set (Eykelbosh et al., 2018). Such partnerships are essential to bringing together the necessary expertise, and although most previous projects have focused on scientific aims, the inclusion of more nonacademic partners (such as public health practitioners) will only strengthen the policy relevance of such projects.

At the NCCEH, we aim to promote policy relevance in research and EIDM by connecting technical and scientific expertise with decision-makers. We do this by participating in numerous public and environmental health networks and initiatives across Canada and by bringing practitioners and researchers into the spotlight through our seminar series and our blog. We have also recently launched a Healthy Built Environment forum, an online national platform for intra- and inter-jurisdictional connections, collaboration, and sharing of best practices and lessons learned. Those seeking collaborative partnerships should reach out to us at contact@ncceh.ca.

\section{Summary}

Intensive efforts in recent years have begun to bear fruit in the form of large data sets that can be used to examine many aspects of Canadian life, including environmental health issues. 
Although good data alone won't get us to great public health policy, data that adequately describe our Canadian communities are immensely valuable within a broader EIDM process that also considers stakeholder engagement, norms and values, and resource constraints, among many other factors. Good data, like those collected by the CCHS and CHMS, can help to identify and understand emerging issues and guide and evaluate interventions. Although barriers to accessing these data exist, Eykelbosh et al. (2018) demonstrated the power of academic and other institutional partnerships to leverage this data in new and useful ways to inform public health policy.

\section{Acknowledgements}

Production of this document has been made possible through a financial contribution from the Public Health Agency of Canada to the National Collaborating Centre for Environmental Health. The author would like to thank Lydia Ma and Tina Chen for their input and edits.

\section{References}

AFN. 2013. First Nations Biomonitoring Initiative: National Results (2011). Ottawa, ON: Assembly of First Nations (AFN). Available at: http://www.afn.ca/uploads/files/afn_fnbi_en_-_2013-06-26.pdf [Accessed October 16, 2017].

Arnason, T., Tanuseputro, P., Tuna, M., Manuel, D. Municipal transportation policy as a population health intervention: estimating the impact of the City of Ottawa Transportation Master Plan on diabetes incidence. Canadian Journal of Public Health. 110(3): 285-93. [Accessed June 01, 2019].

de Sa, E., and Ardern, C. I. 2014. Associations between the built environment, total, recreational, and transit-related physical activity. BMC Public Health. 14(1): 693. Available at: https://doi.org/10. 1186/1471-2458-14-693 [Accessed July 15, 2019].

Eykelbosh, A., Werry, K., and Kosatsky, T. 2018. Leveraging the Canadian Health Measures Survey for environmental health research. Environ Int. 119: 536-543. Available at: https://www. ncbi.nlm.nih.gov/pubmed/30077001 [Accessed July 15, 2019].

Government of Canada. 2019a. Maternal-Infant Research on Environmental Chemicals (MIREC) Study. Available at: https:// www.canada.ca/en/health-canada/services/environmental-workplacehealth/environmental-contaminants/human-biomonitoring- environmental-chemicals/maternal-infant-research-environmentalchemicals-mirec-study.html [accessed 22 July 2019].

Government of Canada. 2012. Monitoring and surveillance activities under Canada's chemicals management plan. Available at: http:// www.chemicalsubstanceschimiques.gc.ca/plan/surveil/index-eng. php [accessed 22 July 2019].

Government of Canada. 2019b. Northern contaminants program. Available at: http://www.science.gc.ca/eic/site/063.nsf/eng/h_7A 463DBA.html [accessed 22 July 2019].

Hajna, S., Ross, N.A., Joseph, L., Harper, S., and Dasgupta, K. 2015. Neighbourhood walkability, daily steps and utilitarian walking in Canadian adults. BMJ Open. 5(11): e008964. Available at: https:// www.ncbi.nlm.nih.gov/pubmed/26603246 [Accessed July 15, 2019].

McMorris, O., Villeneuve, P. J., Su, J., and Jerrett, M. 2015. Urban greenness and physical activity in a national survey of Canadians. Environ Res. 137: 94-100. Available at: http://www.sciencedirect. com/science/article/pii/S0013935114004253 [Accessed July 15, 2019].

National Collaborating Centre for Methods and Tools. 2012. A model for evidence-informed decision-making in public health. [fact sheet]. Available at: https://www.nccmt.ca/uploads/media/media/0001/01/ d9f5cec8637db62f8edda6a6a2551b293a053ede.pdf

Pouliou, T., and Elliott, S. J. 2010. Individual and socio-environmental determinants of overweight and obesity in urban Canada. Health \&Place. 16(2): 389-398. Available at: http://www.sciencedirect. com/science/article/pii/S1353829209001415 [Accessed July 15, 2019].

Statistics Canada. 2019. The Research Data Centres (RDC) network. Available at: https://www.statcan.gc.ca/eng/rdc/network [accessed 18 July 2019].

Thielman, J., Rosella, L., Copes, R., Lebenbaum, M., and Manson, H. 2015. Neighborhood walkability: Differential associations with self-reported transport walking and leisure-time physical activity in Canadian towns and cities of all sizes. Prev Med. 77: 174-180. Available at: http://www.sciencedirect.com/science/article/pii/S009174351500170X [Accessed July 15, 2019].

Ulmer, J. M., Chapman, J. E., Kershaw, S. E., Campbell, M., and Frank, L. D. 2015. Application of an evidence-based tool to evaluate health impacts of changes to the built environment. Can J Public Health. 106(1): ES26. Available at: https://www.ncbi.nlm.nih.gov/pubmed/ 25955544 [Accessed July 15, 2019].

Winters, M., Barnes, R., Venners, S., Ste-Marie, N., McKay, H., Sims-Gould, J., and Ashe, M. C. 2015. Older adults' outdoor walking and the built environment: Does income matter? BMC Public Health. 15(1): 876. Available at: https://doi.org/10.1186/s12889-015-2224-1 [Accessed July 15, 2019]. 